\title{
Superior vena cava replacement combined with venovenous shunt for lung cancer and thymoma: a case series
}

\author{
Wei Dai ${ }^{1 *}$, Jifu Dong ${ }^{2 *}$, Hongwei Zhang ${ }^{2}$, Xiaojun Yang ${ }^{1}$, Qiang Li $^{1}$ \\ ${ }^{1}$ Department of Thoracic Surgery, ${ }^{2}$ Department of Anesthesiology, Sichuan Cancer Hospital \& Institute, Sichuan Cancer Center, School of \\ Medicine, University of Electronic Science and Technology of China, Chengdu 610041, China \\ Contributions: (I) Conception and design: W Dai, J Dong, Q Li; (II) Administrative support: X Yang, Q Li; (III) Provision of study materials or \\ patients: W Dai, J Dong, H Zhang; (IV) Collection and assembly of data: W Dai, J Dong; (V) Data analysis and interpretation: W Dai, J Dong; (VI) \\ Manuscript writing: All authors; (VII) Final approval of manuscript: All authors. \\ *These authors contributed equally to this work. \\ Correspondence to: Qiang Li. Department of Thoracic Surgery, Sichuan Cancer Hospital \& Institute, Sichuan Cancer Center, School of \\ Medicine, University of Electronic Science and Technology of China, No. 55, Section 4, South Renmin Road, Chengdu 610041, China. \\ Email: luckydavii@sina.com.
}

\begin{abstract}
Background: Superior vena cava (SVC) replacement is infrequently performed and technically challenging in low-volume centers. Venovenous shunt (VVS) technique is used to reduce SVC pressure during SVC replacement and has not been well reported. This study aimed to add information on this subject and evaluate the surgical outcomes of patients who underwent SVC replacement combined with VVS in our center.
\end{abstract}

Methods: A retrospective analysis of six patients who underwent SVC replacement combined with VVS from September 2011 to February 2017 was performed. Clinical characteristics, pathological features, operative characteristics, postoperative outcomes, and the survival of the six patients were reviewed.

Results: There were four males and two females with a median age of 44 years (range, 35-69 years). There were three lung cancer patients and three thymoma patients at a stage from IIIA to IVA. Five patients underwent induction therapy. Complete resection was performed on five patients. One patient underwent internal VVS, and the other five patients underwent external VVS. Prosthesis grafts were employed in five cases and autologous pericardium in one case. Three patients underwent single-vein reconstruction, and the other three patients underwent double-vein reconstruction. The median SVC clamping time was 75 minutes. There were no postoperative deaths or major complications. All patients were alive at follow-up, and no thrombosis was found in any of the grafts.

Conclusions: SVC replacement combined with VVS is technically feasible and safe. Although VVS technique is not a must, it may make SVC replacement safer in inexperienced centers. Surgery-based multidisciplinary treatment for selected patients with type T4 lung cancer and SVC involvement or thymoma and SVC involvement may achieve a favorable long-term outcome.

Keywords: Superior vena cava replacement (SVC replacement); venovenous shunt (VVS); vena cava bypass; lung neoplasms; thymoma

Submitted Sep 20, 2017. Accepted for publication Dec 15, 2017.

doi: $10.21037 /$ jtd.2017.12.130

View this article at: http://dx.doi.org/10.21037/jtd.2017.12.130 


\section{Introduction}

Invasion of the superior vena cava (SVC) by lung cancer or thymoma is always considered locally advanced disease. Surgery for these diseases is controversial, due to poor prognosis $(1,2)$. However, with improved surgical techniques and use of neoadjuvant therapy, studies have suggested that SVC resection and reconstruction in locally advanced lung cancer or invasive thymoma with SVC involvement is not only technically feasible, but also has good immediate and long-term outcomes (2-4).

SVC replacement for lung cancer or thymoma is infrequently performed and technically challenging, especially in a center lacking in relevant experience. One of the major obstacles is how to reduce the clamping time of the SVC to reduce neurological complications. Although SVC replacement has been well-documented, there are no data showing which method should be best used to reduce SVC clamping time (2-4). A venovenous shunt (VVS), i.e., a vena cava bypass or caval shunt, as reported by Yoshimura (5), is one of these methods. VVS technique has been reported in published studies involving a few cases (3-6). The indications and details for performing VVS have not been clearly elucidated.

In this study, VVS technique, including external VVS between the internal jugular vein (IJV) and femoral vein and internal VVS between the right innominate vein (RIV) and right atrium (RA) during SVC replacement, is described. The aim of this study is to introduce our experience with patients who undergo SVC replacement combined with VVS, and to analyze the immediate and long-term outcomes of this extended surgery.

\section{Methods}

\section{Patient selection}

From September 2011 to February 2017, six patients underwent SVC replacement combined with VVS, including three lung cancer patients and three thymoma patients, at Sichuan Cancer Hospital. Clinical characteristics, pathological features, operative characteristics, postoperative outcomes, and long-term survival were retrospectively analyzed. The study was approved by the Ethics Committees of Sichuan Cancer Hospital (No. SCCHEC-02-2017-032). Individual consent was obtained from all patients.

\section{Staging and classification}

The pathologic stages of the lung cancer patients were based on the seventh edition of the TNM classification, and thymoma patients were classified according to the Masaoka staging system. The 2004 World Health Organization system for histologic typing of lung tumors and thymic epithelial tumors was used.

\section{VVS technique}

VVS included external VVS between the IJV and femoral vein and internal VVS between the RIV and RA. External VVS was usually instituted before surgery, and internal VVS was instituted during surgery. Another catheter was inserted into the IJV to monitor the central venous pressure when instituting external VVS. The IJV pressure was continuously monitored during surgery and was recorded every 15 min during SVC clamping. Specific operating procedures are presented in Figure 1.

\section{SVC replacement procedures}

SVC replacement included simple SVC resection and reconstruction with a single graft anastomosed between the distal and proximal SVC stumps, and complicated SVC resection and reconstruction involved two grafts anastomosed between the RA and left innominate vein (LIV) and between the RIV and proximal SVC stump. The lengths and diameters of grafts were variable, depending on the degree of SVC involvement and the diameter of the reconstructed vein (SVC or innominate vein). The anastomosis between the graft and vein stump was performed with continuous 4-0 Prolene sutures. Resections and reconstructions were performed with the cross-clamping technique (7). For doublevein reconstruction, the graft was first anastomosed between the right atrial appendage and LIV to reduce the clamping time (8). Part of the SVC replacement procedures is presented in Figure 2.

\section{Intraoperative and postoperative management}

Intravenous sodium heparin $(0.5 \mathrm{mg} / \mathrm{kg})$ was used before SVC clamping. Vasoactive agents were used to obtain an arterial-venous pressure gradient more than $40 \mathrm{mmHg}$ during SVC clamping. Long-term anticoagulation 

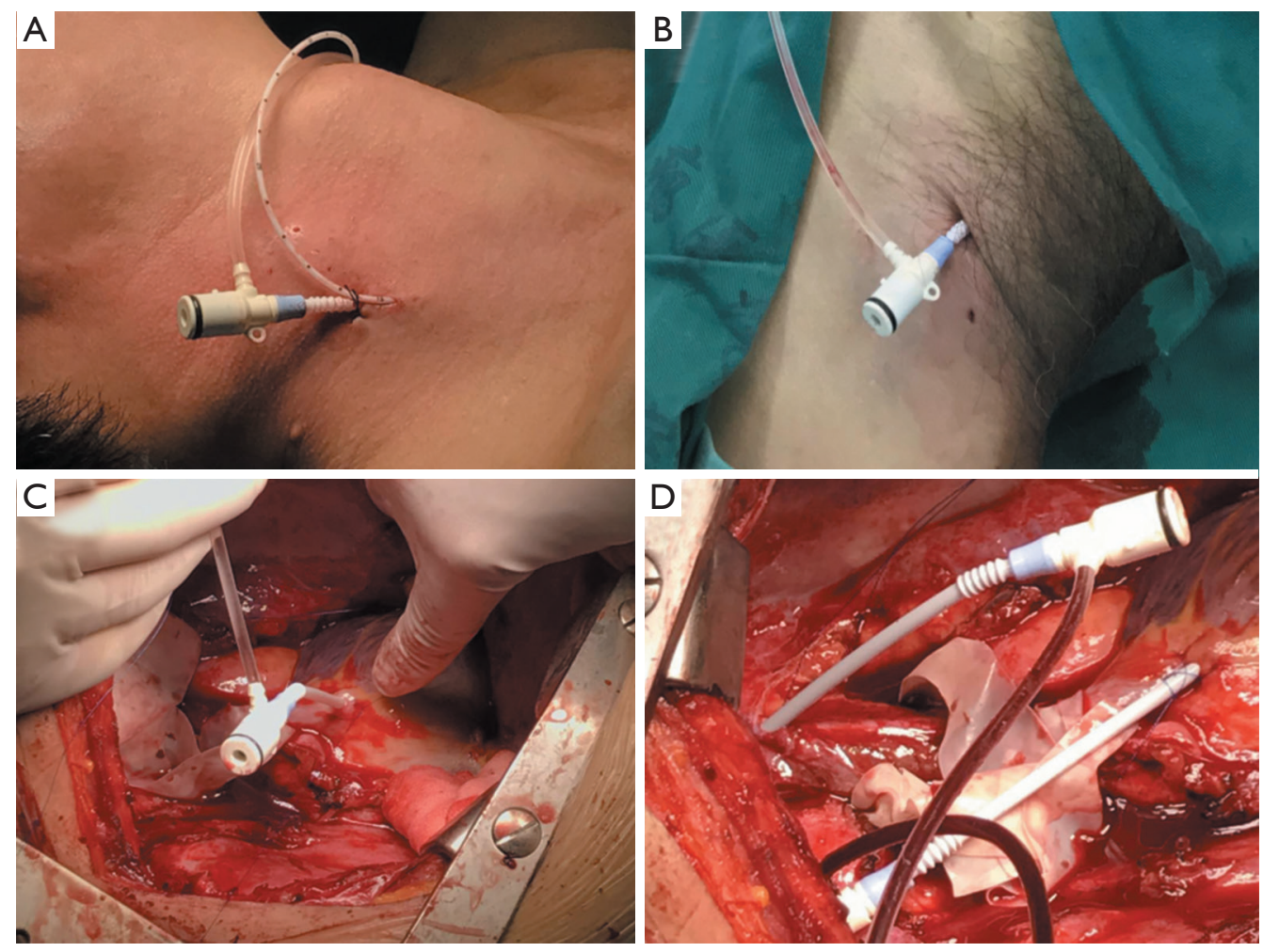

Figure 1 Instituting VVS. (A,B) External VVS was established between the IJV and femoral vein with two venous cannulas (size 8 F) connected by a T-connector. Another catheter was also inserted into the IJV to monitor the central venous pressure; (C,D) internal VVS was established between the RA and RIV using purse-string sutures and two venous cannulas (size 8 F) connected by a T-connector. VVS, venovenous shunt; IJV, internal jugular vein; RIV, right innominate vein; RA, right atrium.

therapy using subcutaneous low-molecular-weight heparin (LMWH) for one month and maintained by oral warfarin was used for patients who underwent SVC replacement with polytetrafluoroethylene (PTFE) Gore-Tex synthetic prostheses. Short-term anticoagulation therapy using subcutaneous LMWH for one month was administered to patients who underwent SVC replacement with autologous pericardium.

\section{Follow-up}

All patients were followed up with chest computed tomography (CT) and patency of the graft was routinely assessed after surgery. Mortality was defined as death occurring within 30 days of surgery or within the initial postoperative hospitalization if this exceeded 30 days. Survival time was defined as the time from surgery to death or last follow-up.

\section{Results}

\section{Clinical and pathological characteristics}

The median age was 44 years (range, 35-69 years). There were three lung cancer patients and three thymoma patients in this study, including two patients with tumor recurrence. No patient presented with SVC syndrome. Five patients underwent preoperative chemotherapy or chemoradiotherapy, and the response was partial response (PR) in three patients and stable disease (SD) in two patients. All tumors in the present cases invaded more than $30 \%$ of the circumference of the SVC. The clinical and pathological characteristics of the six patients are shown in Table 1. The imaging characteristics of patients 1 to 4 are presented in Figure 3.

\section{Operative characteristics}

The median time to institute VVS was $50 \mathrm{~min}$. The 

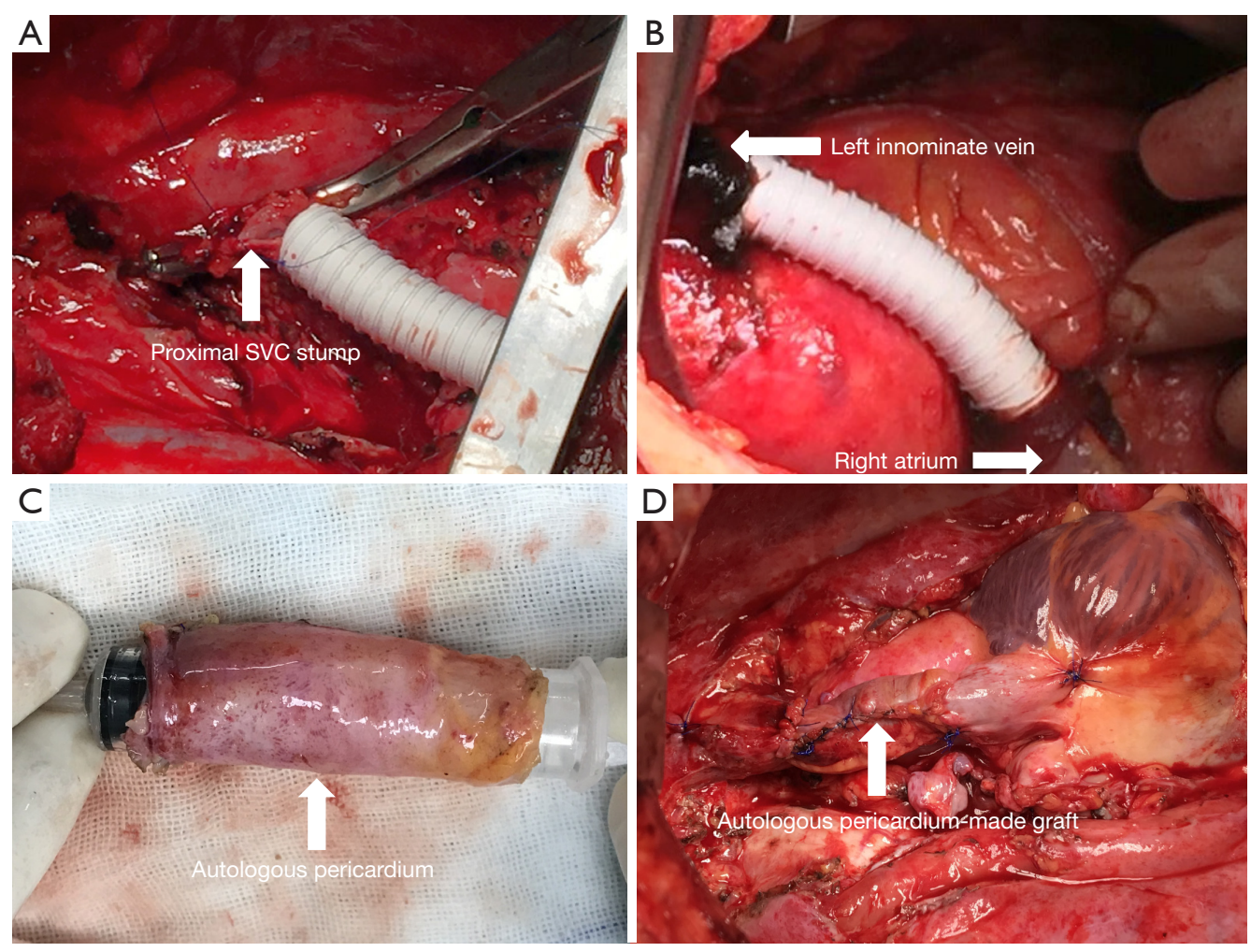

Figure 2 SVC replacement. (A) Reconstruction of SVC with a ringed PTFE prosthesis (size $14 \mathrm{~mm}$ ) between the distal SVC stump and the proximal SVC stump; (B) reconstruction of left SVC system with a PTFE prosthesis (size $12 \mathrm{~mm}$ ) between the RA and LIV; (C) autologous pericardium was made tubular with continuous 4-0 Prolene sutures; (D) reconstruction of SVC with autologous tubular pericardium. SVC, superior vena cava; PTFE, polytetrafluoroethylene; RA, right atrium; LIV, left innominate vein.

Table 1 Clinical and pathological characteristics of six patients

\begin{tabular}{|c|c|c|c|c|c|c|c|}
\hline Pt No. & Age (years), sex & Tumor & Status & Treatment & Response after induction & Histology & Stage \\
\hline 2 & $46, M$ & Lung cancer & Primary & $\mathrm{CT}+\mathrm{S}+\mathrm{CT}$ & PR & Adeno & pT4N2M0, IIIB \\
\hline 3 & $37, \mathrm{~F}$ & Thymoma & Primary & $\mathrm{CRT}+\mathrm{S}+\mathrm{CT}$ & PR & B2 thymoma & III \\
\hline 4 & $42, F$ & Thymoma & Recurrence & $S+S+C R T$ & - & B1 thymoma & III \\
\hline 6 & $69, M$ & Lung cancer & Recurrence & $\mathrm{S}+\mathrm{CRT}+\mathrm{S}+\mathrm{CT}$ & SD & Sq. & pT4NOMO, IIIA \\
\hline
\end{tabular}

Adeno, adenocarcinoma; CRT, chemoradiotherapy; CT, chemotherapy; F, female; M, male; PR, partial response; Pt No., patient number; $\mathrm{RT}$, radiotherapy; S, surgery; Sq., squamous cell carcinoma; SD, stable disease.

median SVC clamping time was $75 \mathrm{~min}$ and the median operative time was $290 \mathrm{~min}$. The median volume of blood loss during the operation was 1,000 mL. Four of the six patients had a blood transfusion, and the median transfusion volume was $800 \mathrm{~mL}$. Complete resection was achieved in five patients and incomplete resection in one patient. The highest mean IJV pressure of patients who received single-vein reconstruction and doublevein reconstruction during SVC clamping when the VVS was open was 15.5 and $47 \mathrm{cmH}_{2} \mathrm{O}$, respectively. The IJV pressure of patient 6 was unavailable because of the internal VVS without IJV pressure monitoring. The IJV 

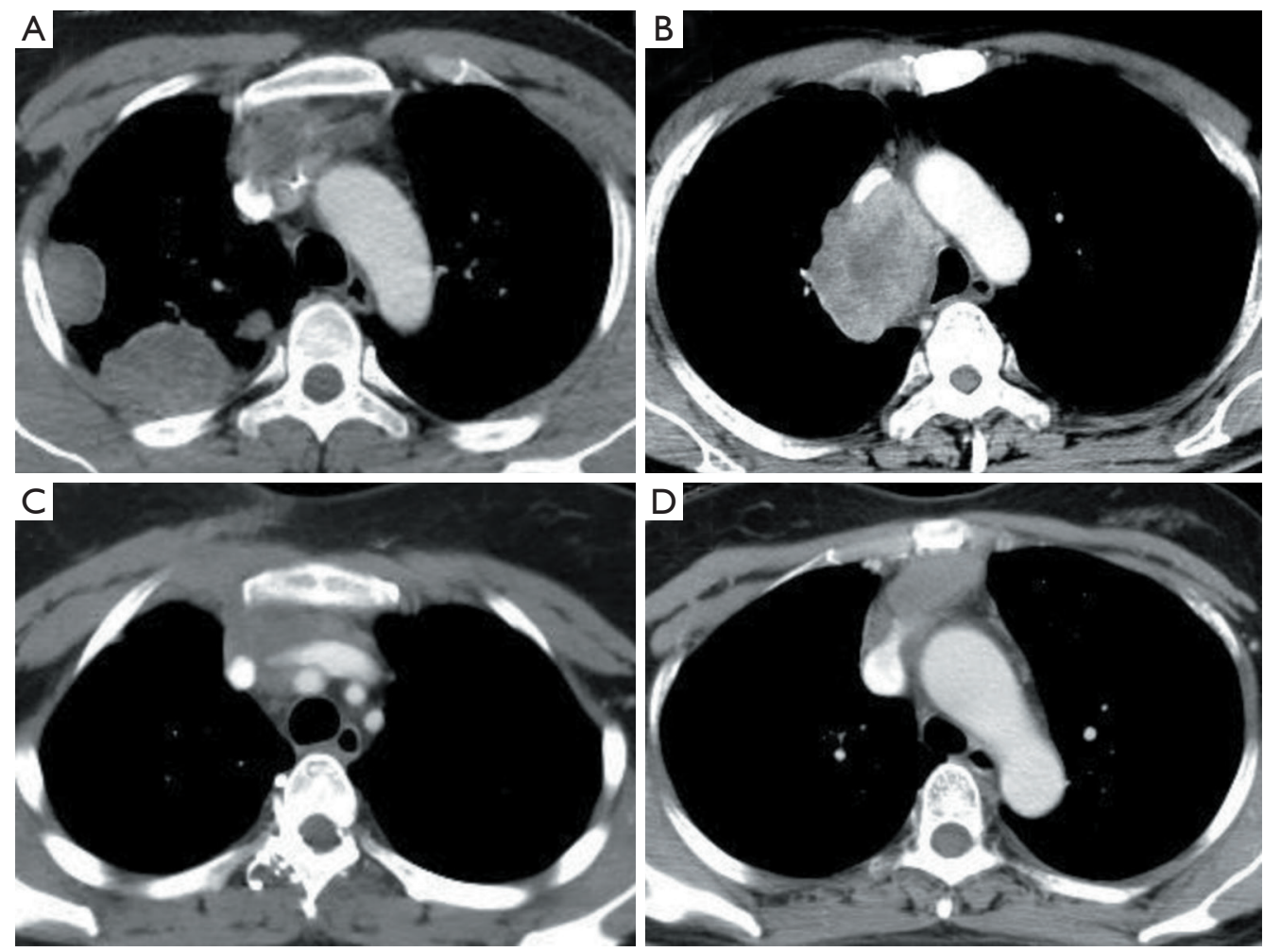

Figure 3 Tumors invading SVC on chest CT. (A) Type B2 thymoma in patient 1; (B) right upper lobe adenocarcinoma in patient 2; (C) type B2 thymoma in patient 3; (D) type B1 thymoma in patient 4. SVC, superior vena cava; CT, computed tomography.

Table 2 Operative characteristics of six patients

\begin{tabular}{|c|c|c|c|c|c|c|c|c|}
\hline Pt No. & Surgery, margin & Approach & SVC graft & VVS & SVC reconstruction & $\mathrm{T}(\min )$ & CT (min) & OT (min) \\
\hline 2 & Lobectomy, R0 & RPLT & Prosthesis & Preo, E & SVC-SVC & 25 & 55 & 210 \\
\hline 3 & ETT, RO & MS & Prosthesis & Preo, E & LIV-RA, RIV-SVC & 60 & 80 & 330 \\
\hline 4 & ETT, RO & MS & Prosthesis & Intra, E & LIV-RA, RIV-SVC & 60 & 100 & 255 \\
\hline 6 & Pneumonectomy, R0 & RPLT & Pericardium & Intra, I & SVC-SVC & 15 & 70 & 260 \\
\hline
\end{tabular}

$\mathrm{CT}$, clamping time of superior vena cava; E, external venovenous shunt between internal jugular vein and femoral vein; ETT, extended resection of thymoma; I, internal venovenous shunt between right innominate vein and right atrium; Intra, intraoperative; LIV, left innominate vein; MS, median sternotomy; OT, operative time; Preo, preoperative; Pt No., patient number; R0, no residual tumor; R2, macroscopic residual tumor; RA, right atrium; RIV, right innominate vein; RPLT, right posterolateral thoracotomy; SVC, superior vena cava; T, time to institute venovenous shunt; VVS, venovenous shunt.

pressures of patients 1 to 5 during SVC clamping when the VVS was closed were also unavailable, because we usually opened the VVS prior to SVC clamping. The operative characteristics for the six patients are shown in Table 2 and Figure 4.

\section{Immediate and long-term outcomes after surgery}

The median length of postoperative hospital stay was 19 days. There were no postoperative major complications or deaths. All patients were alive at follow-up, with a good 
quality of life, although one patient has a recurrent tumor. There was no thrombosis in all grafts. Detailed information about the immediate and long-term outcomes after surgery for all six patients is presented in Table 3 .

\section{Discussion}

SVC replacement is technically challenging and is always performed at a high-volume center. Most of the procedures are performed with SVC blocking. The maximum clamping time for the SVC is still unclear. Some authors stated that the SVC could be safely clamped for $60 \mathrm{~min}$ without neurological complications, and that routine VVS techniques were unnecessary, because a previous chronic SVC system obstruction had led to extensive collateral circulation (9-11). Our center began to perform SVC replacement surgery in 2011. The median SVC clamping time was $75 \mathrm{~min}$, much longer than the time reported in the

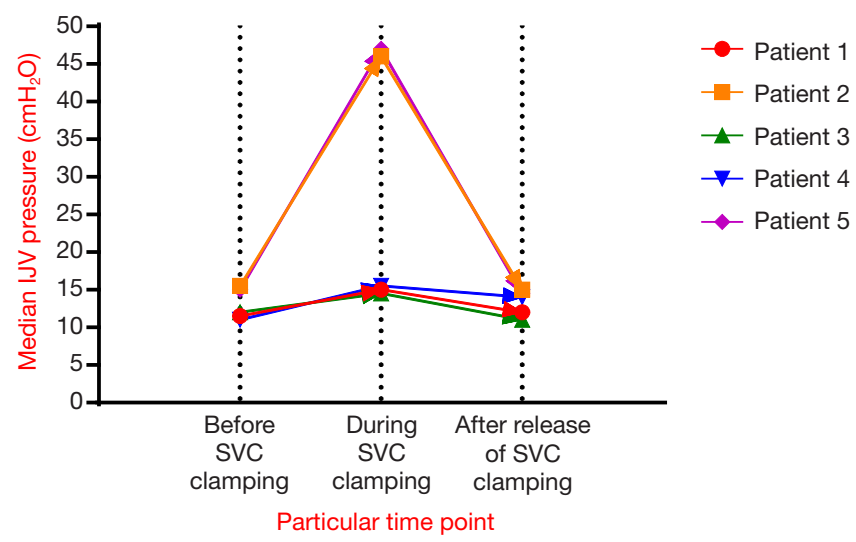

Figure 4 Median IJV pressure of patients 1 to 6 before, during, and after release of SVC clamping. SVC, superior vena cava; IJV, internal jugular vein. literature $(2-4,12,13)$. Because of inexperience and concern about intraoperative emergencies, we often instituted a temporary VVS before surgery and removed it afterwards, to maintain stable hemodynamics during SVC clamping.

In the present series, VVS techniques are used to reduce central venous pressure during SVC clamping. However, the maximum venous pressure that a person can tolerate during SVC remains unknown. Nakahara et al. (14) stated that temporary VVS should be considered if the IJV pressure exceeded $40 \mathrm{cmH}_{2} \mathrm{O}$ during SVC clamping. For other author (15), routine VVS technique was not used because high central venous pressure was well tolerated even if the IJV pressure reached $50 \mathrm{mmHg}\left(68 \mathrm{cmH}_{2} \mathrm{O}\right)$, as long as the arterial-venous pressure gradient was well maintained during the SVC clamping time. In most cases, IJV pressure will rise gradually when the SVC is clamped if there is no VVS. And the lower the IJV pressure is, the safer the surgery will be. As shown in Figure 4, all five patients had a mean IJV pressure less than $50 \mathrm{cmH}_{2} \mathrm{O}$ during SVC clamping when the VVS was open, which could greatly guarantee the safety of surgery particularly when the SVC clamping time was longer than $60 \mathrm{~min}$ in inexperienced centers.

In our study, one patient underwent internal VVS, and the other five underwent external VVS, including three cases of preoperative planned external VVS and two cases of intraoperative, urgent external VVS because of the unexpected finding of SVC involvement. Intraoperative, urgent external VVS prolongs the operative time and increases the risk of postoperative complications. Therefore, this situation should be avoided. Based on this study, our experiences with VVS are summarized as follows. First, instituting external VVS preoperatively is recommended when a center just begins to perform SVC replacement, especially in patients without a chronic SVC system obstruction. Second, preoperative planned external VVS is

Table 3 Immediate and long-term outcomes after surgery in six patients

\begin{tabular}{|c|c|c|c|c|c|c|c|}
\hline Pt No. & $\begin{array}{l}\text { Complications } \\
\text { ( } \geq \text { grade III) }\end{array}$ & Mortality & $\begin{array}{c}\text { Postoperative } \\
\text { hospital stay (days) }\end{array}$ & Recurrence & Outcome & Graft patency & Survival (months) \\
\hline 1 & None & None & 31 & Yes & Alive & Yes & 71 \\
\hline 3 & None & None & 15 & No & Alive & Yes & 31 \\
\hline 4 & None & None & 25 & No & Alive & Yes & 20 \\
\hline 6 & None & None & 11 & No & Alive & Yes & 6 \\
\hline
\end{tabular}

Pt No., patient number. 
preferred, especially when two veins are replaced, because intraoperative catheter insertion for internal VVS will be much more difficult, especially when it is performed at the relatively distal part of the SVC. Third, intraoperative internal VVS can be an alternative in an experienced center, especially when emergencies occur.

For the SVC replacement graft, we always use a PTFE synthetic prosthesis. An autologous pericardium-made vessel was used only in patient 6 , because of the unexpected finding of SVC involvement and the unavailability of a PTFE synthetic prosthesis during surgery (Table 2). As reported in the literature, the advantages of autologous pericardium-made vessels include lower risk of thrombosis, lower risk of infection, and no need for long-term anticoagulant therapy (7). However, autologous pericardium can only be suitable for single-vein replacement due to its limited length. Therefore, a synthetic prosthesis or a heterologous (bovine) prosthesis is mandatory for doublevein replacement of the SVC system $(7,12,16)$.

External VVS, i.e., a shunt from the IJV to femoral vein, was a long bypass in our study, but did not increase the risk of thrombotic complications due to systemic heparinization. On the other hand, utilizing VVS may increase the risk of postoperative bleeding due to systemic heparinization, and thereby increase transfusion requirements and pleural effusion. In this study, the amount of blood loss and blood transfusion volume were greater and the postoperative hospital stays were longer than in common thoracic surgery. However, no bleeding events or thrombotic events occurred. It has also been reported that increased postoperative morbidity and mortality may be associated with induction therapy, second surgery, pneumonectomy, or SVC replacement $(2,4,17,18)$. In our study, five patients received preoperative induction therapy, two underwent second surgery, and two underwent pneumonectomy. However, there were no postoperative deaths or major complications. Therefore, SVC replacement combined with VVS is technically feasible, and has an acceptable perioperative risk $(3,6,13,19)$.

Indications for SVC replacement in our study mainly included resectable tumor on preoperative chest CT, nonprogression response to induction therapy with potential resectability, type A to B2 thymoma, even with stage IVA. Although all the present patients were in stage III or IVA and incomplete resection was achieved in one patient, their survival and quality of life were favorable. This was most probably because the patients were relatively young and strong, and most of the patients (83.3\%) underwent preoperative induction therapy, with down-staging achieved in three patients. According to the literature, the overall 5 -year survival rate of patients with lung cancer and SVC resection can be up to $31 \%$, and is much higher in patients with thymoma and SVC resection $(4,6,20)$. Even for patients with N2 lung cancer, the 5 -year survival can reach $21 \%$, mainly due to induction therapy $(3,4)$. Thus, surgerybased multidisciplinary treatment for selected patients with type T4 lung cancer and SVC involvement or thymoma and SVC involvement may achieve long-term survival.

This study reported our experience of SVC replacement combined with VVS and summarized some indications and details for performing VVS. This study was largely limited by its retrospective nature and small sample size, but the results were in line with previous studies (2-4). Almost all published studies regarding SVC replacement were retrospective, and the sample sizes were also small. Therefore, prospective and larger sample studies are needed to improve the management of SVC replacement and to further evaluate the role of surgery in the treatment of type T4 lung cancer and thymoma with SVC involvement.

In conclusion, SVC replacement combined with VVS is technically feasible and safe. Although VVS technique is not essential, it may make SVC replacement safer in inexperienced centers. Surgery-based multidisciplinary treatment for selected patients with type T4 lung cancer and SVC involvement or thymoma and SVC involvement may achieve a favorable long-term outcome.

\section{Acknowledgements}

Funding: This work was supported by a grant from the Key Research Project of Science and Technology Department of Sichuan Province (2017SZ0013 to Qiang Li).

\section{Footnote}

Conflicts of Interest: The authors have no conflicts of interest to declare.

Ethical Statement: The Ethics Committee of Sichuan Cancer Hospital approved this study (No. SCCHEC-02-2017-032). All patients gave informed consent.

\section{References}

1. Martini N, Yellin A, Ginsberg RJ, et al. Management of non-small cell lung cancer with direct mediastinal involvement. Ann Thorac Surg 1994;58:1447-51. 
2. Spaggiari L, Thomas $\mathrm{P}$, Magdeleinat $\mathrm{P}$, et al. Superior vena cava resection with prosthetic replacement for non-small cell lung cancer: long-term results of a multicentric study. Eur J Cardiothorac Surg 2002;21:1080-6.

3. Shargall $\mathrm{Y}$, de Perrot $M$, Keshavjee S, et al. 15 years single center experience with surgical resection of the superior vena cava for non-small cell lung cancer. Lung Cancer 2004;45:357-63.

4. Spaggiari L, Leo F, Veronesi G, et al. Superior vena cava resection for lung and mediastinal malignancies: a single-center experience with 70 cases. Ann Thorac Surg 2007;83:223-9; discussion 229-30.

5. Yoshimura H, Kazama S, Asari H, et al. Lung cancer involving the superior vena cava: pneumonectomy with concomitant partial resection of superior vena cava. J Thorac Cardiovasc Surg 1979;77:83-6.

6. Thomas P, Magnan PE, Moulin G, et al. Extended operation for lung cancer invading the superior vena cava. Eur J Cardiothorac Surg 1994;8:177-82.

7. Spaggiari L, Galetta D, Veronesi G, et al. Superior vena cava replacement for lung cancer using a heterologous (bovine) prosthesis: preliminary results. J Thorac Cardiovasc Surg 2006;131:490-1.

8. Rossella C, Nazari S. Superior vena cava resection without blood flow interruption. J Thorac Cardiovasc Surg 2007;134:1097-8.

9. Dartevelle PG, Chapelier AR, Pastorino U, et al. Longterm follow-up after prosthetic replacement of the superior vena cava combined with resection of mediastinalpulmonary malignant tumors. J Thorac Cardiovasc Surg 1991;102:259-65.

10. Al-Ayoubi AM, Flores RM. Surgery for lung cancer invading the mediastinum. J Thorac Dis 2016;8:S889-94.

11. Venuta F, Rendina EA. Superior vena cava resection and

Cite this article as: Dai W, Dong J, Zhang H, Yang X, Li Q. Superior vena cava replacement combined with venovenous shunt for lung cancer and thymoma: a case series. J Thorac Dis 2018;10(1):363-370. doi: 10.21037/jtd.2017.12.130 reconstruction. Eur J Cardiothorac Surg 2012;41:1177-8.

12. Ciccone AM, Venuta F, D'Andrilli A, et al. Long-term patency of the stapled bovine pericardial conduit for replacement of the superior vena cava. Eur J Cardiothorac Surg 2011;40:1487-91.

13. Okereke IC, Kesler KA. Superior vena cava and innominate vein reconstruction in thoracic malignancies: single-vein reconstruction. Semin Thorac Cardiovasc Surg 2011;23:323-5.

14. Nakahara K, Ohno K, Mastumura A, et al. Extended operation for lung cancer invading the aortic arch and superior vena cava. J Thorac Cardiovasc Surg 1989;97:428-33.

15. Spaggiari L, Veronesi G, D'Aiuto M, et al. Superior vena cava reconstruction using heterologous pericardial tube after extended resection for lung cancer. Eur J Cardiothorac Surg 2004;26:649-51.

16. Odell DD, Liao K. Superior vena cava and innominate vein reconstruction in thoracic malignancies: doublevein reconstruction. Semin Thorac Cardiovasc Surg 2011;23:326-9.

17. Maugeri L, Corbo GM, Valente S. Postoperative complications after thoracic surgery for lung cancer. Rays 2004;29:465-8.

18. Pei G, Zhou S, Han Y, et al. Risk factors for postoperative complications after lung resection for non-small cell lung cancer in elderly patients at a single institution in China. J Thorac Dis 2014;6:1230-8.

19. Kusumoto H, Shintani Y, Funaki S, et al. Combined resection of great vessels or the heart for non-small lung cancer. Ann Thorac Cardiovasc Surg 2015;21:332-7.

20. Windisch T, Fischer JR, Vega A, et al. Infiltration of the superior vena cava in NSCLC: results of surgical intervention. Pneumologie 2015;69:23-9. 